\title{
Does Interactive Ultrasound Intervention Relieve Minor Depressive Symptoms in Pregnancy? A Randomized Controlled Trial
}

Henrika Peppiina Pulliainen ( $\nabla$ hpherm@utu.fi )

Turun Yliopistollinen Keskussairaala: TYKS Turu yliopistollinen keskussairaala https://orcid.org/00000002-5359-7475

\section{Sari Ahlqvist-Björkroth}

University of Turku, Department of Psychology and Language Pathology, and Department of Clinical Medicine

\section{Eeva Ekholm}

Turun Yliopistollinen Keskussairaala: TYKS Turu yliopistollinen keskussairaala

\section{Research Article}

Keywords: Interactive ultrasound, pregnancy ultrasound, 4D ultrasound, perinatal depression, minor depression, maternal-fetal attachment, mother-infant relationship, psychological support

Posted Date: July 7th, 2021

DOl: https://doi.org/10.21203/rs.3.rs-374219/v1

License: (c) (1) This work is licensed under a Creative Commons Attribution 4.0 International License. Read Full License 


\section{Abstract}

\section{Background}

Perinatal depression, especially minor depression, is common during pregnancy and is likely to continue into the postpartum period. It impairs the mother's health, infant's neurodevelopment, and the motherinfant relationship. Screening for perinatal depression is recommended; however, there is no uniform alignment of how to treat depressive symptoms while simultaneously supporting the mother-infant relationship. Ultrasound screenings might be potential as an intervention method because it has shown to improve maternal-fetal attachment among pregnant women. Our aim is to develop a 4 dimensionalbased (4D) interactive ultrasound intervention and test whether it relieves minor depressive symptoms and improves maternal-fetal attachment. Previous studies show that supporting the mother-infant relationship aids in relieving maternal depression. Until now, few studies have combined pregnancy ultrasound and psychological support.

\section{Methods}

A controlled randomized setting was designed to assess whether interactive 4D-ultrasound intervention would decrease maternal depressive symptoms, strengthen maternal-fetal attachment, and mother-infant relationship. A sonographer and a psychologist specialized in infant mental health conduct the interventions. The focus of the session is to jointly observe the behavior of the fetus according to the mothers' wishes. Altogether, 100 women scoring 10-15 in Edinburgh Pre/-Postnatal Depression Scale (EPDS) and with singleton pregnancy will be recruited using a web-based questionnaire. Half of the participants will be randomized to the intervention group and will receive three interactive ultrasound examinations. The primary outcome is the change in the mean EPDS score. EPDS measurements will be done at three time points: before and after the intervention and four to five months after delivery. The secondary outcomes are maternal representations that will be assessed using the Working Model of the Child Interview (WMCl) and prenatal attachment that will be assessed using the Maternal Antenatal Attachment Scale (MAAS) questionnaire. The postnatal mother-infant interaction will be assessed with the Parent-Child Early Relational Assessment (PCERA) and Maternal Postnatal Attachment Scale (MPAS).

\section{Discussion}

Ultrasound is widely used during pregnancy. The interactive approach is unique and it would be feasible as part of routine screenings and maternity clinic visits. Intervention decreasing depression and simultaneously supporting maternal-fetal attachment could be a valuable addition in treating minor depression among pregnant women.

\section{Trial registration}

Registered on January 5th 2018, ClinicalTrials.gov NCT03424642. https://clinicaltrials.gov/ct2/show/NCT03424642 


\section{Administrative Information}

Title $\{1\} \quad$ Does interactive ultrasound intervention relieve minor depressive symptoms in pregnancy? - A randomized controlled trial

Trial registration Registered on January 5th 2018, ClinicalTrials.gov NCT03424642

$\{2 \mathrm{a}$ and $2 \mathrm{~b}\}$.

Protocol version November 3, 2019. Version 4.

$\{3\}$

Funding $\{4\}$

The study was funded by the Signe and Ane Gyllenberg foundation, the Finnish

Cultural foundation, the Finnish Medical Foundation and State Research

Funding from Hospital Districts of Satakunta and Southwest Finland.

Author details Henrika Pulliainen, MD

$\{5 a\}$

Department of Clinical Medicine, University of Turku, Turku, Finland

hpherm@utu.fi

Sari Ahlqvist-Björkroth, PhD, Psychology

Department of Psychology and Language Pathology, University of Turku, Turku, Finland

sarahl@utu.fi

Eeva Ekholm, MD, PhD, Adjunct Professor, Obstetrics and Gynecology

Department of Obstetrics and Gynecology, Turku University Hospital and University of Turku, Turku, Finland

eeva.ekholm@tyks.fi

Name and

contact

Not applicable. This study received no external funding from a sponsor.

information for

the trial sponsor

$\{5 b\}$

Role of sponsor $\{5 \mathrm{c}\}$

Not applicable. This study received no external funding from a sponsor.

\section{Introduction}

\section{Background and rationale $\{6 \mathrm{a}\}$}

Perinatal depressive symptoms are a significant health problem affecting both mothers' and infants' wellbeing. According to systematic reviews, the prevalence of perinatal depression varies between $8.5 \%$ - and 
$11.9 \%(1,2)$. The incidence in the Finnish population is between $7 \%$ and $-13 \%(3-5)$ and is $14.5 \%$ internationally (6). The use of variable instruments and cut-off points to assess depression partly explain different results (7). In a Finnish cohort sample, $24 \%$ of pregnant women reported moderate levels of depressive symptoms throughout their pregnancy (8). The prevalence of minor depression during pregnancy has not been studied as systematically as major depression, even though minor depression may be more common $(9,10)$. Maternal depressive symptoms during pregnancy tend to remain stable and continue postpartum (1,11-14). Maternal depression has a negative impact on the mother- infant relationship (15-18) predisposing the infant to several adverse neurodevelopmental risks (19-21). A Finnish longitudinal study suggested that the effect of maternal pre- and postnatal depressive symptoms on child neurodevelopment is additive (22). Th fetal effects of medication used during pregnancy should be taken into account favoring nondrug treatments, if possible. For example, selective serotonin reuptake inhibitors (SSRIs) are commonly used antidepressants (23) and fetal prenatal exposure is associated with neonatal maladaptation (24), with increased rates of depression in early adolescence (25).

Maternal perinatal depressive symptoms have an adverse effect on the early mother- infant relationship through three components: pre- and postnatal attachment, mental representations and parent-infant interaction behavior. Maternal prenatal depressive symptoms have a negative association with their attachment to the unborn infant (13,26-29), which, in turn, predict low maternal involvement in the mother- infant interaction $(30,31)$. Prenatal depressive symptoms have also been shown to be a risk factor for suboptimal postnatal maternal attachment $(13,32)$. Pregnant women at the most risk of low levels of attachment were shown to be depressed and also had negative feelings toward the upcoming birth and parenthood (29). Various studies have also shown that prenatal depression distorts the mothers' representation of the infant and their own upcoming motherhood which, in turn, predicts dysfunctional postnatal mother- child interaction (15-17).

The literature has consistently highlighted the importance of identifying women at risk of perinatal depression by screening depressive symptoms and determining effective and individual prevention strategies $(11,17,33,34)$. However, a decrease in a mother's depressive symptoms alone does not necessarily improve parenting or the infant's well-being (35-37). There is some evidence that relationally focused treatments may be more effective than traditional treatments in treating maternal depression $(35,38)$. By supporting the mother- infant relationship, it is possible to decrease maternal depression (13). Thus, early interventions supporting the mother- infant relationship or parenting, should be available in primary health care $(39,40)$ and should start before birth to improve the child outcome $(41)$.

Ultrasound examinations have a beneficial impact on maternal- fetal attachment (42-44) in low-risk pregnancies. Especially, 3D and 4D ultrasounds can enhance mental images about the fetus and increases bonding (45). There are few studies combining pregnancy ultrasound and psychological support (Boukydis, 2006; Jussila, Ekholm, \& Pajulo, 2020; Jussila, Pajulo, \& Ekholm, 2020; H. Pajulo, Pajulo, Jussila, \& Ekholm, 2016; Pulliainen, Niela-Vilén, Ekholm, \& Ahlqvist-Björkroth, 2019) (47-51): these interventions are based on an ultrasound consultation method, where the fetus is observed with motherinitiated interaction (46). Boukydis et al., 2006 studied a small group of women with low-risk pregnancies 
and found participation in ultrasound consultation can improve maternal attachment and decrease general anxiety symptoms. Parenting intervention using interactive 4D ultrasound with mentalization focus among substance- abusing pregnant women showed high attendance to intervention visits compared with routine care (49). Also, the way in which ultrasound examinees comment on and interpret the ultrasound images and baby's actions impacts parents' prenatal mental representations of the baby (52). Routine ultrasound examinations reduce the worried state of mind among pregnant women (53).

Using interactive ultrasound, we performed a qualitative study to map the experiences of women at risk of preterm birth. The pregnant women experienced that the intervention made the fetus more real, strengthening their feelings of attachment to their unborn baby (48). Potentially, the interactive ultrasound intervention may strengthen maternal prenatal attachment and representations and decrease anxiety and depression. Pregnancy ultrasound might have the potential not only as a tool for assessing fetal risks, but also as an instrument in prenatal psychological interventions.

\section{Objectives $\{7\}$}

Aim

The primary aim of the present study is to evaluate, whether interactive ultrasound decreases perinatal depressive symptoms. The secondary aim is to evaluate whether interactive ultrasound improves early maternal attachment and interaction. An additional aim is to document the treatment model and describe it in detail for further use.

The specific hypotheses are as follows:

1. Th intervention decreases prenatal depressive symptoms and the decrease remains postpartum.

a. The mothers in the intervention group have fewer depression symptoms after intervention than before the intervention.

b. The decrease in depression symptoms in the intervention group is greater than in the control group.

2. Intervention improves maternal attachment to the fetus and the infant.

a. The mothers in the intervention group will have a stronger attachment to their baby after the intervention, both during pregnancy and in infancy.

b. The improvement of prenatal attachment from the preintervention to postintervention time in the intervention group is greater when compared with the control group.

c. The mothers in the intervention group have more balanced attachment representation after the intervention than before the intervention.

d. The change will move towards more balanced representations in the intervention group when compared with the control group. 
Moreover, we are exploring the effect of the intervention on maternal psychological distress. However, too little is known about the trajectories of pregnancy related anxiety in low-risk populations to state a hypothesis about this. We will also explore whether minor prenatal depressive symptoms affect the quality of mother- infant interactions. For perinatal outcomes such as preterm birth ( $<37$ gestational weeks, gwks), intrauterine growth restriction (IUGR), and low $(<2.5 \mathrm{~kg})$ birth weight at delivery, the power of the sample is likely to be too low to gain significant differences between groups.

\section{Trial design \{8\}}

The study design is a randomized controlled trial utilizing an intervention group and control group.

\section{Methods: Participants, Interventions And Outcomes Study setting $\{9\}$}

The study is carried at the maternity clinic of Turku University Hospital and Department of Psychology of Turku University in Finland.

\section{Eligibility criteria $\{10\}$}

The inclusion criteria are 1) 10-15 points from the scale (Edinburgh Pre-/Postnatal depression scale), 2) singleton pregnancy, 3 ) over 18 years of age, 4) body mass index under 35 , and 4) fluency in the Finnish language.

The exclusion criteria are psychotic and self-destructive symptoms. The use of antidepressant medication is not an obstacle to participation.

\section{Who will take informed consent? \{26a\}}

An information letter can be found on the project's web- page and is sent to research patients' e-mail addresses after completing the depression screener. Written informed consent are collected from the participants at the beginning of the first study visit by the graduate students of psychology who also conduct the representation interviews.

\section{Additional consent provisions for collection and use of participant data and biological specimens $\{26 \mathrm{~b}\}$}

Not applicable. No biological specimens were collected in this study.

\section{Interventions}

\section{Explanation for the choice of comparators $\{6 \mathrm{~b}\}$}

Real world practice. The Finnish Institution for Health and Welfare recommends that healthy mother clinics screen for depressive symptoms with EPDS in 13-18 gwks. If the total score is 13 or more, the 
pregnant woman should be guided to the doctor's appointment for further assessment. If the total score is 10-12 points, the EPDS should be repeated within 2-4 weeks. In the case of self-destructive thought, immediate help should be organized. Mood should also be assessed by observation and discussion (54).

\section{Intervention description $\{11$ a $\}$}

The method is pregnancy ultrasound combined with an interactive approach. A sonographer and a psychologist specialized in early developmental psychology, conduct the intervention. Three intervention visits are carried out between 25 - and 33 gwks.

The focus of the ultrasound examination is to follow the baby's activities inside the womb, to work with mental images regarding the baby and the pregnancy, and listen to the mother's thoughts and wishes. In the beginning, the sonographer ensures that the pregnant woman understands the images on the screen. Thereafter, the interventionists observe together with the woman what the baby does during the examination. Observations are made on what is seen on the screen and on fetal activities. The interventionists do not make psychological interpretations about the fetus or its behavior. The aim is to facilitate and emphasize pregnant women's own perceptions of the fetus. With 4D- ultrasound, it is possible to see the fetus' face and facial expressions and discuss them with the mother. The intervention visits follow a structured course (Annex 1 . The course of intervention visits). The examination will last approximately 40 minutes, and the women participate alone. The ultrasound examinations are videotaped for later analysis.

The other parent may join the ultrasound examination after the actual intervention to see the baby.

The information about participants' previous psychiatric diagnoses and pregnancy data are collected from electronic medical records. Prenatal care continues as usual.

\section{Criteria for discontinuing or modifying allocated interventions $\{11 \mathrm{~b}\}$}

No modifications are made. All participants receive the same intervention dose; three interactive ultrasound examinations lasting approximately 40 minutes each.

\section{Strategies to improve adherence to interventions $\{11 \mathrm{c}\}$}

There are three intervention teams each comprising a psychologist and an obstetrician, a specialist in maternal-fetal medicine. All the interventionists receive training in the interactive approach. The structure of each session is described, and key elects of the sessions are listed. Intervention teams gather together frequently to review video recorded intervention sessions to enhance adherence to the course of the intervention visits.

\section{Relevant concomitant care permitted or prohibited during the trial $\{11 d\}$}


Prenatal care and possible maternity clinic visits continue as usual. Also, other support or therapeutic treatments are allowed, but they are documented and controlled for the analysis.

\section{Provisions for post-trial care $\{30\}$}

No.

\section{Outcomes $\{12\}$}

The primary outcome of the study is perinatal depressive symptoms analyzed as a change in the EPDSmean score from before the intervention to after the intervention. The secondary outcomes are maternalinfant-relationship- related outcomes; pre- and postnatal attachment, prenatal representations, maternal pregnancy- related psychological distress, and mother-infant interaction. The secondary outcomes will be analyzed with both quantitative and qualitative methods.

\section{Participant timeline $\{13\}$}

Annex 2. Research chart.

\section{Sample size $\{14\}$}

The study aims to compare changes in EPDS scores between two independent groups. A mean decrease of three points in the EPDS score was considered clinically relevant and used for power calculations. A standard deviation of 3.5 points in EPDS used in power calculations was based on earlier studies in the Finnish population showing an SD of 3.7-4 (17). Power analysis using a statistical power of $80 \%$ and a significance level of 0.05 (two-tailed) showed that 50 subjects are needed in both groups. Because of an estimated attrition of 10\%, 60 subjects for both groups will be enrolled. Randomization was performed with random permuted blocks, using block size 10. The SAS ${ }^{\circ}$ was used to generate the randomization (Version 9.4 for Windows).

\section{Recruitment \{15\}}

Pregnant women with depressive symptoms are recruited by an advertisement shared in social media by the Department of Obstetrics and Gynecology of Turku University Hospital and by brochures distributed at routine screening scans. The advertisement presents a link to a web page, where the interested can fill in an EPDS- questionnaire and leave their contact information. The researcher contacts those who have EPDS-score of 10-15 points for recruitment and ensures that those interested meet the inclusion criteria. The potential participants are interviewed with SCID (Structured Clinical Interview for DSM (Diagnostic and Statistical Manual of Mental Disorders) -III- R), and the appointment to the first WMCl-interview is booked. The researcher will also contact the women whose EPDS score is $\geq 16$ to refer them to care.

Study data are collected and managed using REDCap electronic data capture tools hosted at the University of Turku $(55,56)$. REDCap (Research Electronic Data Capture) is a secure, web-based software platform designed to support data capture for research studies, providing 1) an intuitive interface for validated data capture; 2) audit trails for tracking data manipulation and export procedures; 3) automated 
export procedures for seamless data downloads to common statistical packages; and 4) procedures for data integration and interoperability with external sources.

Due to the Covid19 pandemic, recruitment was suspended from March 16th to September 22nd in 2020.

\section{Assignment of interventions: allocation Sequence generation $\{16 \mathrm{a}\}$}

Randomization for intervention and control groups was performed with random permuted blocks, using block size 10. SAS ${ }^{\circledR}$ was used to generate the randomization (Version 9.4 for Windows, SAS Institute Inc., Cary, NC, USA), and programming was done by biostatistician.

\section{Concealment mechanism $\{16 \mathrm{~b}\}$}

The randomization list is kept in a locked place to which only the study group has access. After the first representation interview, the interviewer looks from a separate randomization list to which group the participant is assigned and reveals it to the participant. Two graduate students of psychology carry out the interviews. The interviews are scheduled by the gestational weeks of the participants.

\section{Implementation $\{16 \mathrm{c}\}$}

A biostatistician carried out the allocation sequence before the start of the present randomized study. A doctorate researcher contacts potential participants to recruit them, ensures that they meet the inclusion criteria, performs an SCID -interview, and makes an appointment to first the WMCl-interview. The graduate students of psychology perform the first $\mathrm{WMCl}$ - interview, after which they look from the allocation sequence into which group the participant will be allocated. There are two graduate students working on the project simultaneously.

\section{Assignment of interventions: Blinding Who will be blinded $\{17$ a $\}$}

The representation $(\mathrm{WMCl})$ interview data are analyzed in a blinded fashion by two trained coders with an appropriate level of reliability. One coder evaluates the preintervention interviews, and another evaluates the postintervention interviews. The coders are not completely blinded, because one of the coders is a part of the intervention team. To avoid bias of the coder who also belongs to the intervention team only codes the preintervention interviews. The second coder, who is not a part of the intervention team, codes the postintervention interviews. The participants may talk about the intervention visits during their postintervention interview revealing their group allocation.

Mother- infant interaction PCERA video recordings are analyzed by two trained coders who have received an appropriate level of reliability. One coder functions as a main coder, doing $100 \%$ of the codings; this coder is not completely blind to the sample because she has interviewed some participants and therefore, may know their study group status. The other coder is the reliability coder, who does $25 \%$ of the codings, 
which overlaps with the main coder. However, the person who is conducting the reliability coding is completely blind for the sample.

\section{Procedure for unblinding if needed $\{17 \mathrm{~b}\}$}

Not applicable. See 17a.

\section{Data collection and management}

\section{Plans for assessment and collection of outcomes $\{18 \mathrm{a}\}$}

The outcomes of the study are assessed and collected using mixed methods approach. The patients are recruited utilizing social media. Data are collected by videotaping traditional appointments and with electronic questionnaires. Because of the COVID19 -pandemic, the interviews may be carried out with remote connections.

\section{Data collection measures}

\section{The Edinburg Pre-/Postnatal Depression Scale (EPDS)}

Depressive symptoms are assessed using the EPDS. The EPDS is a reliable, validated and internationally accepted 10-item self-rating instrument for screening depressive symptoms, with the score ranging from 0 to 30 points $(7,57)$. For minor depression, the lowest cut- off score is 10 points $(7,57,58)$. The cut offscore for major depression varies from 13 points $(59,60)$ to 15 points $(60)$. In Finnish cohort, the cut- off score of $\geq 12$ points was used for probable depression (61). The higher the score, the higher the probability of clinical depression.

\section{The Structured Clinical Interview for DSM (SCID)}

The is a valid, diagnostic, and widely used semi-structured interview designed to evaluate psychopathology categorically. It can be used to assess the severity of dimensions of depression, for example (62). The interview is reliable in clinical screening as well as for research purposes, and is applicable by telephone (63). Only the depression module of the SCID is used in the present study. The SCID interview is chosen to verify clinical depression and its severity.

\section{Pregnancy-Related Anxiety Questionnaire Revised (PRAQ- R2)}

Pregnancy specific anxiety is assessed with a validated and abbreviated version of Pregnancy Related Anxiety Questionnaire Revised (PRAQ -R2) $(64,65)$, which is a shortened version of the 34-item PRAQ (66). PRAQ-R2 is suitable for use in pregnant women regardless, of parity. It is a 10 -item self-report scale that consists of three subscales: (1) fear of giving birth, (2) fear of bearing physically or mentally handicapped children and (3) concern about one's appearance. Each item score ranges from 1 (definitely not true) to 5 (definitely true). A higher score represents a higher level of anxiety.

\section{Symptoms Checklist 90 (SCL-90)}


General anxiety symptoms are measured with the anxiety subscale of the Symptoms Checklist 90 (SCL90), which is a valid and reliable 10-item self-report, with each item rating from 0-5 (67). It can be used in both clinical and research settings (68).

\section{Prenatal maternal relationship to the unborn infant: \\ The Maternal Antenatal Attachment Scale (MAAS)}

Prenatal emotional attachment is measured using the Maternal Antenatal Attachment Scale (MAAS), which is a validated 19-item instrument designed to evaluate prenatal attachment to the unborn child in the dimensions of "quality of attachment" (10 items: experiences of closeness, tenderness, pleasure in interaction, distress at fantasized loss and the conceptualizing of the fetus as a "little person") and "intensity/time spent in attachment mode" (8 items: the amount of time spent thinking about, talking to, dreaming about or palpating the fetus, quality of involvement and intensity of preoccupation). One item (feeling whether the baby is dependent on the mother for its well-being) is not included in either dimension but is included in the global score. A high combined score indicates a strong attachment and relationship to the fetus $(28,69,70)$.

\section{The Working Model of the Child Interview (WMCl)}

Prenatal attachment representations are assessed with the $\mathrm{WMCl}$ (71). This semi- structured interview systematically explores parents' thoughts, feelings and perceptions of their expected baby and the relationship with their baby. We use a prenatal version of the $\mathrm{WMCl}(72)$. The interviews are conducted and recorded in a laboratory setting.

The recorded representational narratives will be evaluated according to the $\mathrm{WMCl}$ coding manual (Zeanah, Benoit, 1996). First, the narrative is evaluated with six qualitative scales (Richness of Perceptions, Openness to Change, Intensity of Involvement, Coherence, Caregiving Sensitivity, and Acceptance) and two content scales (Infant Difficulty and Fear of Infant's Safety). Thereafter, the narratives are categorized into balanced, disengaged and distorted representations. Disengaged and distorted representations can also be classified as nonbalanced representations. Trained coders will perform the coding.

\section{Postnatal mother- infant relationship:}

\section{The Maternal Postnatal Attachment Scale (MPAS)}

Postnatal emotional attachment is measured using the (MPAS), which is a validated 19-item postpartum counterpart of Condon's (70) MAAS. It measures the mother's reported feelings about her infant by three factors: (1) quality of bonding, (2) absence of hostility, and (3) pleasure in interaction (74). A higher score indicates a more adaptive mother- infant bonding style (74).

\section{The Parent- Child Early Relational Assessment (PCERA)}


The mother- infant interaction is assessed with a widely used (Lotzin et al., 2015) video- observation method, Parent- Child Early Relational Assessment (PCERA; Clark, 1985). It consists of 65 independent items: 29 parental, 28 child, and eight dyadic items. Ratings are based on five minutes of video-recorded parent- child interactions. The items are scored from 1 to 5 , so that scores 1 and 2 represent an area of concern, 3 is an area of some concern, and 4 and 5 are an area of strength. The method has good factorial validity and evidence for convergent and discriminant validity as well (Clark, 1999; Lotzin et al., 2015).

In the current study, mother- infant interaction is video-recorded during a free-play session. The video recordings are performed in a laboratory setting to standardize the environment. The PCERA situations will be analyzed according to the manual (Clark, 1985) by two trained coders.

\section{Plans to promote participant retention and complete follow- up $\{18 b\}$}

Participant retention can be strengthened by phone calls or SMS as needed.

\section{Data management $\{19\}$}

Data file description is required by the Ethics Committee of the Hospital District of Southwest Finland in order to receive research permission. The participants' files are preserved separately from the research data in a secure place. The participants will get an ID code, that will be used for data storage and analysis. The research data are pseudoanonymized: the recruitment log of the participants is used to undo the ID codes and is kept separately from the data files. Form data are stored in REDCap (56), which, in turn, is stored on a secure network drive of the University of Turku. Videotapes are stored in a secure online storage of the University of Turku.

The University of Turku automatically provides copies of the research data into three independent servers when the data are stored. The servers also function as a data backup system. Only the members of the study group have access to the data. Access to the storage is controlled by the principal investigator of the study. Access also requires a personal username issued by the University of Turku. The allowance to access the data is issued only for the active research period. The principal investigator will keep a record of those who have active access to the data. All the data will be disposed of 10 years after the last publication.

\section{Confidentiality $\{27\}$}

See above.

\section{Plans for collection, laboratory evaluation and storage of biological specimens for genetic or molecular analysis in this trial/future use $\{33\}$.}




\section{Statistical methods}

\section{Statistical methods for primary and secondary outcomes $\{20$ a $\}$}

The categorical variables of the study will be characterized using frequencies and percentages. Continuous variables with means and standard deviations or medians with range and/or 25th and 75th percentiles will be used. Age will be summarized with the mean and range.

If assumptions for parametric analyses are met, mean change of EPDS score over time will be analyzed using a linear mixed modeling approach for repeated measures. Then, the time point will be considered as a within-factor and the treatment intervention as a between factor-effect. Also, the effect of the participants' background variables' parity, age, antidepressant medication, care contacts within healthcare, and so forth, will be tested. Time point $x$ intervention interaction will describe whether the mean changes over time are different between the groups. The covariance structure of the unstructured (UN) and compound symmetry (CS) will be tested. The differences (together with $95 \% \mathrm{Cl}$ ) between the groups will also be estimated at each time point, as well as within group changes. Transformation for the EPDS or for other outcomes can be performed before analyses, if needed. The assumptions of parametric tests will be checked using studentized residuals for justification of the analyses. Other numerical variables, mean change of MAAS, MPAS, SCL-90, and PRAQ-R2 score over time, will be analyzed in a similar way.

P-values less than 0.05 will be considered statistically significant (two-tailed). Statistical analyses will be performed using SAS System for Windows, Version 9.4 or later (SAS Institute Inc., Cary, NC).

\section{Interim analyses $\{21 \mathrm{~b}\}$}

Not applicable. No interim analyses will be done in this study.

\section{Methods for additional analyses (e.g. subgroup analyses) \{20b\}}

The sample size and the subgroups are small. Preliminary analyses will be made to see if there are statistically significant differences within and between the study groups.

\section{Methods in analysis to handle protocol non-adherence and any statistical methods to handle missing data $\{20 \mathrm{c}\}$}

Because of relatively small sample size, imputation will not be carried out. 


\section{Plans to give access to the full protocol, participant level- data and statistical code $\{31 \mathrm{c}\}$}

Metadata can be published on the web-page of the project without compromising the participants' data protection. Metadata includes information on what kind of data has been collected, at what time, from whom and how. Requests for collaboration are encouraged.

\section{Oversight and monitoring}

\section{Composition of the coordinating centre and trial steering committee $\{5 d\}$}

Not applicable. The authors of the protocol coordinate this study.

\section{Composition of the data monitoring committee, its role and reporting structure $\{21 \mathrm{a}\}$}

The study group is independent.

\section{Adverse event reporting and harms $\{22\}$}

The doctoral student of the research team continously follows up the information collected from the questionnaires and contacts the women, who have conceded self-destructive thoughts or plans or have score $\geq 16$ in EPDS. All the women scoring between 10 and 15 are also contacted because they are recruited to the study.

\section{Frequency and plans for auditing trial conduct $\{23\}$}

Not applicable. The study group meet regularly. The quality research visits are monitored from the video recordings.

Plans for communicating important protocol amendments to relevant parties (e.g. trial participants, ethical committees) $\{25\}$

Any changes in study protocol must be accepted by the Ethics Committee of the Hospital District of Southwest Finland before enforcement.

\section{Dissemination plans $\{31 \mathrm{a}\}$}

The results will be published in an international open access publication and will be linked to the web page of the project, with a plain language summary in Finnish. The questionnaire results or interview analyses are not reported to the participants personally. 
Maternal depressive symptoms remain stable during pregnancy and into the postpartum period. They predispose the infant to adverse neurodevelopment, that is an increased risk to emotional, behavioral, and cognitive problems (19-21). Prenatal depression is relatively common, and subclinical symptoms of depression and experience of distress are especially challenging to detect (11). Early screening for perinatal depression is widely recommended $(33,54,78)$. Few tools to alleviate depressive symptoms are available in maternity care, so a referral to mental health professionals is often required (54). However, there is a risk that pregnant women identified as suffering from minor depressive symptoms do not receive adequate support and treatment. This may have far-reaching consequences on both the infant's and mother's health $(33,79,80)$. Nonmedical therapies should be preferred because prenatal use of antidepressant medication- SSRIs- may have negative effects on infants' health $(24,25)$. An ultrasound assisted intervention could be a low- threshold type of to support and easily feasible in maternity care.

Pregnancy ultrasound is widely and routinely used in screenings and in risk pregnancies as a diagnostic tool. According to a current review, fetal ultrasound is considered a promising method to support maternal- fetal attachment (81). In general, pregnant women are motivated to attend ultrasound examinations (53). In our previous qualitative study, we also found that pregnant women with an imminent preterm birth experienced the interactive ultrasound as supporting their active and equivalent participation, which also enabled them to consider the fetus as a real person, strengthening the mental image of the baby (48). Psychotherapeutic and psychosocial interventions are effective in treating perinatal depression (82), but require special expertise and resources. Therefore, an ultrasound examination used in an interactive manner may be a potential and cost-effective way to support pregnant women with minor or moderate depressive symptoms.

The strengths of our study are the mixed methods and longitudinal approach that continues postpartum. Further, the multiprofessional approach enables the support of pregnant women in various ways. A weakness in our randomized controlled trial may be that the comparison group is also taking part in the representation interviews, which can be experienced as an intervention itself.

Our aim is to develop an intervention for pregnant women with depressive symptoms using 4D ultrasound in an interactive way and to do so in a randomized and controlled setting. The interactive approach is unique and could be integrated into maternity care with only a moderate investment. The core elements of the intervention- listening to the mother, personalizing the fetus, and giving emotional support- can easily be reached during routine screening and maternity clinic visits. This intervention could serve as a means for maternity care to help women suffering from minor perinatal depression.

\section{Trial}

The protocol version number on March 22nd 2021 is four. The first participant was recruited on September 19th 2018. By March 22nd 2021, 52 participants have been recruited. The recruitment will be completed within two years. 


\section{Abbreviations}

4D 4 dimensional

3D 3 dimensional

EPDS Edinburgh Pre-/Postanatal Depression Scale

WMCI Working Model of the Child Interview

PCERA Parent- Child Early Relational Assessment

MAAS Maternal Antenatal Attachment Scale

MPAS Maternal Postnatal Attachment Scale

PRAQ-R2 Pregnancy Related Anxiety Questionnaire Revised

SCL-90 Symptoms Checklist 90

SCID Structured Clinical Interview for DSM

DSM Diagnostic and Statistical Manual of Mental Disorders

IUGR intrauterine growth restriction

gwks gestational weeks

\section{Declarations}

\section{Acknowledgements}

The authors thank Riikka Korja, Associate Professor in Psychology and Docent in Developmental Psychology, for valuable comments in planning this intervention. We also thank Eliisa Löyttyniemi, Biostatician at University of Turku, for statistical consultation. The authors want to acknowledge Kirsi Rinne, PhD in Gynecology and Obsterics, and Nina Ginström, Master in Psychology, for collaboration in conducting the interventions.

\section{Authors' contributions}

EE and SAB designed the study and supervised the writing of the protocol. HP has recruited the participants and collected the questionnaire data. $S A B$ has organized the interviews and video recordings and trained of the coders. EE, SAB, and HP conduct the interactive ultrasound examinations. EL did the statistical analyses. All authors participated in the writing and approved the final manuscript.

\section{Funding}


The study was funded by the Signe and Ane Gyllenberg foundation, the Finnish Cultural foundation, the Finnish Medical Foundation and State Research Funding from Hospital Districts of Satakunta and Southwest Finland. All the scholarships have provided financial support enabling research work occasionally full-time in addition to clinical work. The funding bodies have no role in the design of the study and collection, analysis and interpretation of Data and in writing the manuscript.

\section{Availability of data and material}

Metadata will be published on the project's web page.

\section{Ethics approval and consent to participate}

The study was approved by the Ethics Committee of the Hospital District of Southwest Finland, case number 95/1801/2017. Written informed consent was obtained from the participants.

\section{Consent for publication}

Consent for publication not applicable.

\section{Competing interests}

The authors declare that they have no competing interests.

\section{Authors' information (optional)}

\section{References}

1. Woody CA, Ferrari AJ, Siskind DJ, Whiteford HA, Harris MG. A systematic review and meta-regression of the prevalence and incidence of perinatal depression. Journal of Affective Disorders. 2017.

2. Gavin NI, Gaynes BN, Lohr KN, Meltzer-Brody S, Gartlehner G, Swinson T. Perinatal depression: A systematic review of prevalence and incidence. Obstetrics and Gynecology. 2005.

3. Tammentie T, Tarkka MT, Åstedt-Kurk P, Paavilainen E. Sociodemographic factors of families related to postnatal depressive symptoms of mothers. Int J Nurs Pract. 2002;

4. Pajulo M, Savonlahti E, Sourander A, Helenius H, Piha J. Antenatal depression, substance dependency and social support. J Affect Disord. 2001;

5. Ahlqvist-Björkroth S, Vaarno J, Junttila N, Pajulo $M$, Räihä H, Niinikoski H, et al. Initiation and exclusivity of breastfeeding: Association with mothers' and fathers' prenatal and postnatal depression and marital distress. Acta Obstet Gynecol Scand. 2016;

6. Gaynes BN, Gavin N, Meltzer-Brody S, Lohr KN, Swinson T, Gartlehner G, et al. Perinatal depression: prevalence, screening accuracy, and screening outcomes. Evidence report/technology assessment (Summary). 2005. 
7. Bergink V, Kooistra L, Lambregtse-van den Berg MP, Wijnen H, Bunevicius R, van Baar A, et al. Validation of the Edinburgh Depression Scale during pregnancy. J Psychosom Res. 2011;

8. Korja R, Nolvi S, Kataja EL, Scheinin N, Junttila N, Lahtinen H, et al. The courses of maternal and paternal depressive and anxiety symptoms during the prenatal period in the finnbrain birth cohort study. PLoS One. 2018;

9. Ashley JM, Harper BD, Arms-Chavez CJ, LoBello SG. Estimated prevalence of antenatal depression in the US population. Arch Womens Ment Health. 2016;

10. Marchesi C, Bertoni S, Maggini C. Major and minor depression in pregnancy. Obstet Gynecol. 2009;

11. Eastwood J, Ogbo FA, Hendry A, Noble J, Page A. The impact of antenatal depression on perinatal outcomes in Australian women. PLoS One. 2017;

12. Ogbo FA, Eastwood J, Hendry A, Jalaludin B, Agho KE, Barnett B, et al. Determinants of antenatal depression and postnatal depression in Australia. BMC Psychiatry. 2018;

13. Goecke TW, Voigt F, Faschingbauer F, Spangler G, Beckmann MW, Beetz A. The association of prenatal attachment and perinatal factors with pre- and postpartum depression in first-time mothers. Arch Gynecol Obstet. 2012;286(2):309-16.

14. Leigh B, Milgrom J. Risk factors for antenatal depression, postnatal depression and parenting stress. BMC Psychiatry. 2008;

15. Dayton CJ, Levendosky AA, Davidson WS, Bogat GA. The child as held in the mind of the mother: The influence of prenatal maternal representations on parenting behaviors. Infant Ment Health J. 2010;

16. Tambelli R, Odorisio F, Lucarelli L. Prenatal and postnatal maternal representations in nonrisk and atrisk parenting: Exploring the influences on mother-infant feeding interactions. Infant Ment Health J. 2014;

17. Ahlqvist-Björkroth S, Korja R, Junttila N, Savonlahti E, Pajulo M, Räihä H, et al. Mothers' and fathers' prenatal representations in relation to marital distress and depressive symptoms. Infant Ment Health J. 2016;37(4):388-400.

18. Hazell Raine K, Nath S, Howard LM, Cockshaw W, Boyce P, Sawyer E, et al. Associations between prenatal maternal mental health indices and mother-infant relationship quality 6 to 18 months' postpartum: A systematic review. Infant Ment Health J. 2020;

19. Talge NM, Neal C, Glover V. Antenatal maternal stress and long-term effects on child neurodevelopment: How and why? Journal of Child Psychology and Psychiatry and Allied Disciplines. 2007.

20. Van Den Bergh BRH, Mulder EJH, Mennes M, Glover V. Antenatal maternal anxiety and stress and the neurobehavioural development of the fetus and child: Links and possible mechanisms. A review. In: Neuroscience and Biobehavioral Reviews. 2005.

21. Kingston D, Tough S, Whitfield H. Prenatal and postpartum maternal psychological distress and infant development: A systematic review. Child Psychiatry and Human Development. 2012. 
22. Tuovinen S, Lahti-Pulkkinen M, Girchenko P, Lipsanen J, Lahti J, Heinonen K, et al. Maternal depressive symptoms during and after pregnancy and child developmental milestones. Depress Anxiety. 2018;

23. Malm H, Artama M, Brown AS, Gissler M, Gyllenberg D, Hinkka-Yli-Salomäki S, et al. Infant and childhood neurodevelopmental outcomes following prenatal exposure to selective serotonin reuptake inhibitors: Overview and design of a Finnish Register-Based Study (FinESSI). BMC Psychiatry. 2012;

24. Malm H, Sourander A, Gissler M, Gyllenberg D, Hinkka-Yli-Salomäki S, McKeague IW, et al. Pregnancy complications following prenatal exposure to SSRIs or maternal psychiatric disorders: Results from population-based national register data. Am J Psychiatry. 2015;

25. Malm H, Brown AS, Gissler M, Gyllenberg D, Hinkka-Yli-Salomäki S, McKeague IW, et al. Gestational Exposure to Selective Serotonin Reuptake Inhibitors and Offspring Psychiatric Disorders: A National Register-Based Study. J Am Acad Child Adolesc Psychiatry. 2016;

26. McFarland J, Salisbury AL, Battle CL, Hawes K, Halloran K, Lester BM. Major depressive disorder during pregnancy and emotional attachment to the fetus. Arch Womens Ment Health. 2011;

27. Seimyr L, Sjögren B, Welles-Nyström B, Nissen E. Antenatal maternal depressive mood and parentalfetal attachment at the end of pregnancy. Arch Womens Ment Health. 2009;

28. Condon JT, Corkindale $\mathrm{C}$. The correlates of antenatal attachment in pregnant women. $\mathrm{Br} \mathrm{J}$ Med Psychol. 1997;

29. Rubertsson C, Pallant JF, Sydsjö G, Haines HM, Hildingsson I. Maternal depressive symptoms have a negative impact on prenatal attachment - findings from a Swedish community sample. J Reprod Infant Psychol. 2015;33(2):153-64.

30. Siddiqui A, Hägglöf B. Does maternal prenatal attachment predict postnatal mother-infant interaction? Early Hum Dev. 2000;59(1):13-25.

31. Dubber S, Reck C, Müller M, Gawlik S. Postpartum bonding: the role of perinatal depression, anxiety and maternal-fetal bonding during pregnancy. Arch Womens Ment Health. 2015;

32. Cuijlits I, van de Wetering AP, Endendijk JJ, van Baar AL, Potharst ES, Pop VJM. Risk and protective factors for pre- and postnatal bonding. Infant Ment Health J. 2019;

33. Khanlari S, Barnett Am B, Ogbo FA, Eastwood J. Re-examination of perinatal mental health policy frameworks for women signalling distress on the Edinburgh Postnatal Depression Scale (EPDS) completed during their antenatal booking-in consultation: A call for population health intervention. BMC Pregnancy and Childbirth. 2019.

34. Freeman MP. Perinatal Depression: Recommendations for Prevention and the Challenges of Implementation. JAMA - Journal of the American Medical Association. 2019.

35. Forman DR, O'Hara MW, Stuart S, Gorman LL, Larsen KE, Coy KC. Effective treatment for postpartum depression is not sufficient to improve the developing mother-child relationship. Dev Psychopathol. 2007;

36. Tsivos ZL, Calam R, Sanders MR, Wittkowski A. Interventions for postnatal depression assessing the mother-infant relationship and child developmental outcomes: A systematic review. International 
Journal of Women's Health. 2015.

37. Nylen KJ, Moran TE, Franklin CL, O’Hara MW. Maternal depression: A review of relevant treatment approaches for mothers and infants. Infant Mental Health Journal. 2006.

38. Clark R, Tluczek A, Brown R. A mother-infant therapy group model for postpartum depression. Infant Ment Health J. 2008;

39. Lefkovics E, Baji I, Rigó J. Impact of maternal depression on pregnancies and on early attachment. Infant Ment Health J. 2014;

40. Salo SJ, Flykt M, Mäkelä J, Biringen Z, Kalland M, Pajulo M, et al. The effectiveness of Nurture and Play: A mentalisation-based parenting group intervention for prenatally depressed mothers. Prim Heal Care Res Dev. 2019;

41. Glover V, Capron L. Prenatal parenting. Current Opinion in Psychology. 2017.

42. Sedgmen B, McMahon C, Cairns D, Benzie RJ, Woodfield RL. The impact of two-dimensional versus three-dimensional ultrasound exposure on maternal-fetal attachment and maternal health behavior in pregnancy. Ultrasound Obstet Gynecol. 2006;27(3):245-51.

43. Reading AE, Campbell S, Cox DN, Sledmere CM. Health beliefs and health care behaviour in pregnancy. Psychol Med [Internet]. 1982;12(2):379-83. Available from: http://www.ncbi.nlm.nih.gov/pubmed/7100360

44. de Jong-Pleij EAP, Ribbert LSM, Pistorius LR, Tromp E, Mulder EJH, Bilardo CM. Three-dimensional ultrasound and maternal bonding, a third trimester study and a review. Prenat Diagn. 2013;33(1):818.

45. Wenstrom KD. Preexamination and postexamination assessment of parental-fetal bonding in patients undergoing 3-/4-dimensional obstetric ultrasonography: Commentary. Vol. 62, Obstetrical and Gynecological Survey. 2007. p. 165-6.

46. Boukydis Z. Ultrasound consultation to reduce risk and increase resilience in pregnancy. In: Annals of the New York Academy of Sciences. 2006. p. 268-71.

47. Pajulo H, Pajulo M, Jussila H, Ekholm E. Substance-abusing pregnant women: prenatal intervention using ultrasound consultation and mentalization to enhance the mother-child relationship and reduce substance use. Infant Ment Health J. 2016;37(4):317-34.

48. Pulliainen $\mathrm{H}$, Niela-Vilén $\mathrm{H}$, Ekholm E, Ahlqvist-Björkroth S. Experiences of interactive ultrasound examination among women at risk of preterm birth: A qualitative study. BMC Pregnancy Childbirth. 2019;

49. Jussila H, Pajulo M, Ekholm E. A Novel 4D Ultrasound Parenting Intervention for Substance Using Pregnant Women in Finland: Participation in Obstetric Care, Fetal Drug Exposure, and Perinatal Outcomes in a Randomized Controlled Trial. Matern Child Health J. 2020;

50. Jussila H, Ekholm E, Pajulo M. A New Parental Mentalization Focused Ultrasound Intervention for Substance Using Pregnant Women. Effect on Self-reported Prenatal Mental Health, Attachment and Mentalization in a Randomized and Controlled Trial. Int J Ment Health Addict. 2020; 
51. Boukydis CFZ, Treadwell MC, Delaney-Black V, Boyes K, King M, Robinson T, et al. Women's Responses to Ultrasound Examinations During Routine Screens in an Obstetric Clinic. J Ultrasound Med [Internet]. 2006;25(6):721-8. Available from: http://doi.wiley.com/10.7863/jum.2006.25.6.721

52. Walsh TB. Your baby is so happy, active, uncooperative: How prenatal care providers contribute to parents' mental representations of the baby. Midwifery. 2020;

53. Ekelin M, Svalenius EC, Larsson AK, Nyberg P, Maršál K, Dykes AK. Parental expectations, experiences and reactions, sense of coherence and grade of anxiety related to routine ultrasound examination with normal findings during pregnancy. Prenat Diagn. 2009;

54. Klemetti R, Hakulinen-Viitanen T. Äitiysneuvolaopas. Suosituksia äitiysneuvolatoimintaan. [Internet]. THL, Helsinki, Finland. 2013. p. 1-412. Available from: THL_OPA2013_029_verkko.pdf

55. Harris PA, Taylor R, Thielke R, Payne J, Gonzalez N, Conde JG. Research electronic data capture (REDCap)-A metadata-driven methodology and workflow process for providing translational research informatics support. J Biomed Inform. 2009;

56. Harris PA, Taylor R, Minor BL, Elliott V, Fernandez M, O’Neal L, et al. The REDCap consortium: Building an international community of software platform partners. Journal of Biomedical Informatics. 2019.

57. Cox JL, Holden JM, Sagovsky R. Detection of Postnatal Depression: Development of the 10-item Edinburgh Postnatal Depression scale. Br J Psychiatry. 1987;

58. Bunevicius A, Kusminskas L, Pop VJ, Pedersen CA, Bunevicius R. Screening for antenatal depression with the Edinburgh Depression Scale. J Psychosom Obstet Gynecol. 2009;

59. Rubertsson C, Börjesson K, Berglund A, Josefsson A, Sydsjö G. The Swedish validation of Edinburgh Postnatal Depression Scale (EPDS) during pregnancy. Nordic Journal of Psychiatry. 2011.

60. Matthey S, Henshaw C, Elliott S, Barnett B. Variability in use of cut-off scores and formats on the Edinburgh Postnatal Depression Scale - Implications for clinical and research practice. Arch Womens Ment Health. 2006;

61. Karlsson L, Tolvanen M, Scheinin NM, Uusitupa HM, Korja R, Ekholm E, et al. Cohort Profile: The FinnBrain Birth Cohort Study (FinnBrain). Int J Epidemiol. 2018;

62. Shankman SA, Funkhouser CJ, Klein DN, Davila J, Lerner D, Hee D. Reliability and validity of severity dimensions of psychopathology assessed using the Structured Clinical Interview for DSM-5 (SCID). Int J Methods Psychiatr Res. 2018;

63. Osório FL, Loureiro SR, Hallak JEC, Machado-de-Sousa JP, Ushirohira JM, Baes CVW, et al. Clinical validity and intrarater and test-retest reliability of the Structured Clinical Interview for DSM-5 Clinician Version (SCID-5-CV). Psychiatry Clin Neurosci. 2019;

64. Huizink AC, Mulder EJH, Robles De Medina PG, Visser GHA, Buitelaar JK. Is pregnancy anxiety a distinctive syndrome? Early Hum Dev. 2004;

65. Huizink AC, Delforterie MJ, Scheinin NM, Tolvanen M, Karlsson L, Karlsson H. Adaption of pregnancy anxiety questionnaire-revised for all pregnant women regardless of parity: PRAQ-R2. Arch Womens Ment Health. 2016; 
66. Van den Bergh BR. The influence of maternal emotions during pregnancy on fetal and neonatal behavior. J Prenat Perinat Psychol Heal. 1990;

67. Derogatis LR, Lipman RS, Covi L. SCL-90: an outpatient psychiatric rating scale-preliminary report. Psychopharmacol Bull. 1973;

68. Holi MM, Sammallahti PR, Aalberg VA. A Finnish validation study of the SCL-90. Acta Psychiatr Scand. 1998;

69. MS C. Development of a tool for the measurement of maternal attachment during pregnancy. Nurs Res. 1981;

70. Condon JT. The assessment of antenatal emotional attachment: Development of a questionnaire instrument. Br J Med Psychol. 1993;

71. Zeanah CH, Benoit D, Hirshberg L, Barton ML, Regan C. Mothers' representations of their infants are concordant with infant attachment classifications. Dev Issues Psychiatry Psychol. 1994;

72. Benoit $\mathrm{D}$, Parker $\mathrm{KCH}$, Zeanah $\mathrm{CH}$. Mothers' representations of their infants assessed prenatally: Stability and association with infants' attachment classifications. J Child Psychol Psychiatry Allied Discip. 1997;

73. Zeanah C.H.Benoit D. HL. Working Model of the Child Interview coding manual. 1996.

74. Condon JT, Corkindale CJ. The assessment of parent-to-infant attachment: Development of a selfreport questionnaire instrument. J Reprod Infant Psychol. 1998;

75. Clark R. The parent-child early relational assessment: A factorial validity study. Educ Psychol Meas. 1999;

76. Lotzin A, Lu X, Kriston L, Schiborr J, Musal T, Romer G, et al. Observational Tools for Measuring Parent-Infant Interaction: A Systematic Review. Clinical Child and Family Psychology Review. 2015.

77. Clark R. The parent-child early relational assessment: Instrument and manual. Madison Univ Wisconsin Med Sch Dep Psychiatry. 1985;

78. Le Strat Y, Dubertret C, Le Foll B. Prevalence and correlates of major depressive episode in pregnant and postpartum women in the United States. J Affect Disord. 2011;

79. Cox EQ, Sowa NA, Meltzer-Brody SE, Gaynes BN. The perinatal depression treatment cascade: Baby steps toward improving outcomes. Journal of Clinical Psychiatry. 2016.

80. Letourneau NL, Dennis CL, Cosic N, Linder J. The effect of perinatal depression treatment for mothers on parenting and child development: A systematic review. Depression and Anxiety. 2017.

81. Borg Cunen N, Jomeen J, Borg Xuereb R, Poat A. A narrative review of interventions addressing the parental-fetal relationship. Women and Birth. 2017.

82. Sockol LE, Epperson CN, Barber JP. A meta-analysis of treatments for perinatal depression. Clinical Psychology Review. 2011.

\section{Supplementary Files}


This is a list of supplementary files associated with this preprint. Click to download.

- Annex1.Thecourseofinterventionvisits170321.docx

- Annex2.Researchchart210221.docx 